\title{
FOLIC ACID TREATMENT OF ZAMBIAN CHILDREN WITH MODERATE TO SEVERE MALARIA ANEMIA
}

\author{
MODEST MULENGA, PHIDELIS MALUNGA, STEVE BENNETT, PHILIP THUMA, CAROLINE SHULMAN, \\ KATHERINE FIELDING, AND BRIAN GREENWOOD* \\ Macha Malaria Research Institute, Macha, Zambia; Tropical Diseases Research Centre, Ndola, Zambia; London School of Hygiene \\ and Tropical Medicine, London, United Kingdom
}

\begin{abstract}
Whether administration of folic acid to children with malaria anemia is helpful is controversial. Therefore, we conducted a randomised, placebo-controlled trial of 14 days of treatment with folic acid (1 mg/d) in Zambian children with malaria anemia treated with either sulfadoxine/pyrimethamine (SP) or atovaquone/proguanil (AP). Among children who received SP, the prevalence of parasitemia was higher in children treated with folic acid than among those given placebo at days 3, 7, and 14 after the start of treatment, and the difference at day 3 was statistically significant $(P=0.013)$. Folic acid treatment had no effect on parasitemia in children treated with AP. Administration of folic acid led to a small increase in packed cell volume over that seen in the placebo group at days 14 and 28 after the start of treatment.
\end{abstract}

\section{INTRODUCTION}

Anemia is a serious complication of Plasmodium falciparum infection and a major cause of morbidity and mortality in young Zambian children. ${ }^{1}$ The pathogenesis of malarial anemia is complex, but red cell destruction, either directly by the parasite or as a result of an immune attack, is considered to be the major cause. However, underlying iron and/or folic acid deficiencies may be aggravating factors. Treatment of malarial anemia requires an effective anti-malarial drug to eliminate parasitemia, but supportive therapies such as hematinics and blood transfusion may also be needed to enhance hematological recovery. The high prevalence of HIV infection in Zambia increases the risk of blood transfusion.

Recent studies have provided some evidence in favor of iron supplementation after anti-malarial treatment of children with malaria anemia, ${ }^{2}$ but the value of treatment with folic acid remains controversial. ${ }^{3}$ It has been suggested that, in the presence of a poor dietary intake of folic acid, the rapid erythroid hyperplasia that occurs after hemolysis can cause depletion of folate stores, which may, in turn, lead to delayed hematological recovery. ${ }^{4}$ However, some studies have shown that folate deficiency is uncommon in African children with malarial anemia, ${ }^{5}$ and folate supplementation may not be necessary for their hematological recovery.

Sulfadoxine/pyrimethamine (SP) is still the first line treatment of uncomplicated malaria in many parts of Africa, although this situation is changing. Concern has been expressed that co-administration of folic acid with SP could antagonize the anti-parasitic activity of this drug. Exogenous folate can antagonize anti-folate anti-malarial drugs such as SP and proguanil in vitro, ${ }^{6}$ and it has been suggested that high red cell folate concentrations favor parasite growth ${ }^{7}$ and that folate deficiency may be protective. ${ }^{8}$ Three previous studies in African children give some support to the view that the efficacy of SP my be compromised by the co-administration of folic acid. In The Gambia, folic acid given to children with uncomplicated malaria at a dose of $5 \mathrm{mg} / \mathrm{d}$ for 28 days had a significant effect on the parasitological response to treatment with

\footnotetext{
* Address correspondence to Brian Greenwood, Department of Infectious and Tropical Diseases, London School of Hygiene and Tropical Medicine, Keppel St., London WC1E 7HT, UK. E-mail: brian.greenwood@1shtm.ac.uk
}

SP. ${ }^{9}$ In Kenya, patients with uncomplicated malaria (mean age, 80 months) were treated with SP and iron and allocated randomly to receive folic acid ( 2.5 or $5 \mathrm{mg}$ according to age) or not for 30 days. Survival analysis showed delayed parasite clearance in the patients who received folic acid, which was most marked in the first week after the start of treatment, but no effect on clinical recovery. ${ }^{10}$ In Malawi, children with uncomplicated falciparum malaria who failed treatment with SP had a small, but significantly higher, mean blood folate concentration than children who had an adequate clinical and parasitological recovery. ${ }^{11}$

Before the start of this trial, folic acid was given routinely to children with malarial anemia seen at Macha Mission Hospital, Zambia, but the staff was uncertain of its benefit. To determine this, we undertook a randomized, placebocontrolled trial of treatment with folic acid in children with moderate to severe malarial anemia (SMA). Patients in the trial were randomized to receive SP or atovaquone/proguanil (AP), using a factorial design. The results of the comparison in the efficacies of SP and AP in the treatment of malaria anemia will be presented elsewhere (Mulenga M, Malunga P, Bennett S, Thuma P, Shulman C, Fielding K, Alloueche A, Greenwood B, unpublished data). This trial was undertaken to help the Malarone Donation Program decide how to use most effectively the one million doses of AP offered for use in Africa by GlaxoSmithKline. This proved to be difficult to do, and the donation policy has not been pursued.

\section{MATERIALS AND METHODS}

Study design. The study compared children with moderate to severe malaria anemia treated with SP or AP and folic acid or placebo using a factorial design. The study protocol was approved by the Tropical Diseases Research Center, Ndola, and the London School of Hygiene and Tropical Medicine Ethics Committees. The study was monitored by an independent Data and Safety Monitoring Board (DSMB).

Study site and duration. The study was carried out in the 40-bed pediatric ward at Macha Mission Hospital (MMH), located in the southern province of Zambia. Malaria transmission in the area is seasonal, occurring between December and May. Children were recruited in two consecutive malaria transmission seasons: December 2000 to June 2001 (Phase 1) and February to June 2002 (Phase 2). 
Study subjects. Children 6-119 months of age who presented to the outpatient department were eligible for enrollment if they met the following inclusion criteria: a blood film positive for asexual forms of $P$. falciparum at a density of $50-500,000$ parasites $/ \mu \mathrm{L}$; the presence of fever $\left(\geq 37.5^{\circ} \mathrm{C}\right)$ or a history of fever in the preceding 24 hours; a packed cell volume (PCV) of 9-21\%; a body weight of $5 \mathrm{~kg}$ or greater. After review by the DSMB during the course of the trial, the PCV cut-off was raised from $9 \%$ to $12 \%$. Exclusion criteria were an inability to take oral medication; an inability to sit or stand unsupported; respiratory distress or other symptoms and signs of severe malaria as defined by the World Health Organization (WHO), ${ }^{12}$ with the exception of anemia or hyperparasitemia; serious concurrent illnesses such as severe malnutrition or tuberculosis; receipt of an anti-malarial other than chloroquine in the previous 2 weeks; an allergy to antifolates; receipt of folic acid in the previous week; and blood transfusion in the previous month.

Sample size. It was assumed that the treatment failure rate within 14 days of the start of treatment in children who received SP or AP would be about $10 \%$. It was hypothesized that co-administration of folic acid would increase this to $20 \%$. To detect this difference with a one-sided, type 1 error of 0.05 (it was considered highly improbable that folic acid would increase the efficacy of SP or AP) and $80 \%$ power, 305 children were required in each study arm.

Randomization. Before the start of the trial, a computergenerated randomization list was prepared by the DSMB's statistician to assign treatments in equal numbers (in blocks of 16) to either AP or SP with or without folic acid supplementation. Folic acid or placebo was given only to children who had a PCV greater than $15 \%$, because of concern over a possible, serious adverse interaction between SP and folate in severely anemic children. All drugs were individually randomized and pre-packed by two people unconnected with the trial. The DSMB's statistician kept one copy of the randomization code and the senior hospital pharmacist kept the other copy at the study site.

Study drugs and dose. Study children were admitted to the hospital for 3 days and received, under supervision, either AP (Malarone; GlaxoSmithKline, Brentford, UK) at a dose of approximately $17 \mathrm{mg}$ atovaquone/7 $\mathrm{mg}$ proguanil/kg orally once daily for 3 days or SP (Cosmos Pharmaceuticals, Nairobi, Kenya) at a dose of approximately $25 \mathrm{mg} / \mathrm{kg}$ sulfadoxine on one occasion. The solubility and drug content of the SP tablets was validated by high performance liquid chromatography. Folic acid (Cosmos Pharmaceuticals) was administered as a 1-mg tablet given once daily for 14 days, the first four doses under supervision. Concomitant medications, such as paracetamol for fever, were documented. However, children who needed treatment with anti-microbial agents known to have anti-malarial properties were excluded. Any patient with clinical or parasitological failure or who was judged by the clinician in charge to be developing danger signs of complicated malaria was given quinine in an initial dose of 20 $\mathrm{mg} / \mathrm{kg}$ intravenously or $10 \mathrm{mg} / \mathrm{kg}$ orally, depending on their clinical condition. Oral quinine was continued at a dose of 10 $\mathrm{mg} / \mathrm{kg}$ every 8 hours for a further 6 days. Patients received blood transfusion if their PCV fell below 9\%, regardless of clinical status, if they developed respiratory distress, or if they developed extreme lethargy or were not improving.

On discharge, parents were given sufficient folic acid tab- lets or placebo for a further 10 days of treatment, and parents were instructed to give the tablets to children at home.

Clinical management. Children were managed according to the WHO guidelines for treatment of complicated malaria. A clinician assessed symptoms once daily or more frequently if the patient was very ill, and vital signs were measured every 4 hours. A clinician determined the prevalence and severity of any adverse events (AEs) that occurred during admission or follow-up. Patients were discharged on the third day provided that they had normal vital signs and were able to take oral medication. After discharge, children were followed up at home on days 7, 14, and 28. At each visit, a study clinician assessed the child's recent symptoms, recorded any medications taken since discharge, and carried out a physical examination. A clinical officer followed up absentee children at the home the same day or the next day.

Laboratory investigations. All laboratory investigations were carried out without knowledge of the patient's treatment group. At days $0,3,7,14,21$, and 28, blood smears for parasite counts were prepared, stained for 15 minutes in $10 \%$ Giemsa stain, and examined by two experienced microscopists working independently. Parasites were counted against 200 white blood cells, and parasite density was calculated on the basis of measured white cell count. A blood smear was declared negative if no parasites were seen after examination of 100 high power fields. If the parasite density showed a difference of one logarithm or greater between readers, M.M. adjudicated the final result. MSP2 typing was used to differentiate re-infections from recrudescences. ${ }^{13}$

PCV was measured at days $0,3,7,14$, and 28 after the start of study treatment. At days 0,14 , and 28, a full blood count was done using an automated counter (Cobas-Micros; Hoffmann-La Roche, Basel, Switzerland). A reticulocyte index was determined on fresh blood samples obtained on days 0 and 14. Blood glucose concentrations were measured using a glucometer (Glucotrend; Hoffmann-La Roche) before study drug intake and at anytime during the study if hypoglycemia was suspected. At days 0 and 14 , serum specimens were collected, stored at $-20^{\circ} \mathrm{C}$, and at the end of the study, transported to the Human Nutrition Research Laboratories of the Medical Research Council, Cambridge, UK, where folate concentrations were analyzed using the IMx system folate assay (Abbott Laboratories, Abbott Park, IL).

Trial endpoints. The primary trial endpoint was treatment failure, defined as the occurrence of any one of the following within 14 days of the start of treatment: a need for escape medication (quinine); a need for blood transfusion; failure to increase PCV to greater than $21 \%$; or death. Additional endpoints were the presence or absence of parasitemia at days 3 , 7, 14, and 28 after the start of treatment, the mean PCV at days 7,14, and 28 after treatment, the mean corpuscular volume (MCV) 7 and 14 days after treatment, and the mean reticulocyte count 14 days after the start of treatment. AEs were defined as any symptoms or signs that developed or increased in severity after treatment within a period of 7 days of the initiation of trial treatment. However, serious AEs were monitored up to day 28.

Statistical methods. Data were double-entered, validated (EPI-INFO version 6.04b; CDC), and analyzed using STATA version 7. Primary outcomes were analyzed by intent-to-treat. Thus, all children who received the first dose of study medication were analyzed. $\chi^{2}$ test or Fisher exact test, or multi- 
variate analyses by unconditional logistic regression, were used to compare binary outcomes such as treatment failure. The likelihood ratio test was used to compare different multivariate models. Means of continuous outcomes such as PCV and parasite density were compared using the Student $t$ test and multiple regression analysis, following normalization when necessary. Mann-Whitney $U$ test was used to compare continuous outcomes that were not normally distributed such as serum folate concentrations.

An analytical plan was prepared before the randomization code was broken. An initial analysis studied whether there was any interaction between the two arms of the trial, the comparison between two anti-malarials, and that between folic acid treatment and placebo administration. No interaction was found; therefore, for clarity, the folic acid supplementation and the anti-malarial treatment components of the trial are being presented in separate papers.

\section{RESULTS}

One hundred eighty-three (72\%) of the 255 patients enrolled into the main study who had a PCV greater than $15 \%$ were randomized to receive either folic acid or placebo. Two additional patients with a PCV of $15 \%$ inadvertently received folic acid and have been included in the analysis. Forty-seven children were allocated to the folic acid plus AP group, 45 to the folic acid plus SP group, 47 to the placebo plus AP group, and 46 to the placebo plus SP group. The distribution of baseline characteristics among children in the folic acid and placebo groups was similar (Table 1).

Treatment failure. Eighteen patients failed treatment: 7 $(8 \%)$ in the folic acid group and $11(12 \%)$ in the placebo group. The effect of folic acid supplementation on treatment failure was not significant (odds ratio [OR]: 0.62; 95\% confidence interval $[\mathrm{CI}]: 0.19,1.86 ; P=0.35)$. Treatment failures were distributed as follows: three patients in the folic acid group and four in the placebo group received escape medication, two patients in the folic acid group and four in the placebo group received a blood transfusion, and one patient in the folic acid group and two patients in the placebo group died within 14 days of the start of study treatment. All three deaths occurred within 48 hours of admission; hypoglycemia was implicated in two of the deaths. Among patients who

TABLE 1

Baseline variables in the folic acid treatment and placebo groups

\begin{tabular}{lcc}
\hline \multicolumn{1}{c}{ Baseline characteristics } & $\begin{array}{c}\text { Folic acid } \\
(n=92)\end{array}$ & $\begin{array}{c}\text { Placebo } \\
(n=93)\end{array}$ \\
\hline Antipyretic intake last 24 hours \% $(n)$ & $68(62)$ & $63(59)$ \\
Sex: female \% $(n)$ & $52(47)$ & $52(48)$ \\
Breast-fed (\%) & $51(46)$ & $56(52)$ \\
Dill-Glazko test positive \% $(n)$ & $65(43 / 66)$ & $56(40 / 71)$ \\
Referral from Health Centre \% $(n)$ & $44(40)$ & $43(40)$ \\
Traditional medication \% $(n)$ & $38(35)$ & $41(38)$ \\
Bed net use \% $(n)$ & $10(9)$ & $8 \%(7)$ \\
Hookworm ova \% $(n)$ & $4(2 / 53)$ & $0(0 / 53)$ \\
Packed cell volume (\%) [mean (SD)] & $18.2(1.4)$ & $18.0(1.3)$ \\
White cell count $\times 10 \% / L$ [mean (SD)] & $11.1(5.8)$ & $11.3(5.7)$ \\
Mean corpuscular volume (fl) & $78.9(10.6)$ & $79.5(11.7)$ \\
[mean (SD)] & $1.7(1.5)$ & $1.8(1.5)$ \\
Reticulocyte index [mean (SD)] &
\end{tabular}

received SP, there were 6 failures among 45 children who received folic acid and 9 among 46 who received placebo $(P=0.42)$, whereas among patients who received AP, there were two failures in each group.

Parasite clearance. The proportions of children in whom asexual malaria parasites were detected and mean parasite densities in positive blood smears at days 3, 7,14, and 28 after the start of treatment are shown in Table 2. During the first few days after the start of treatment, more children in the folate than in the placebo group were parasitemic, and at day 3 , the difference between groups was statistically significant $(P<0.02)$. Differences between the groups were not apparent on days 7,14 , or 28 . Further analysis showed that, among children who received folic acid, there was a significant interaction between parasitological findings and the anti-malarial drug used (logistic model, likelihood ratio test $P$ for interaction $=0.05)$. Among children who received AP, there were no significant differences in parasite prevalence between children who received folic acid and those who did not. Thus, at day 3 , the prevalence of parasitemia in children who had received folic acid was 4/46 compared with $3 / 45$ in those who had received placebo. Only three children in either group were parasitemic after day 3 . However, among children who received $\mathrm{SP}$, the prevalence of parasitemia was higher at days 3,7 , and 14 in children who had received folic acid than in those who had received placebo, and the difference between groups at day 3 was statistically significant (Table 3 ). No significant differences in the number of patients with gametocytes were observed between groups on any day of follow-up (data not shown).

Hematological indexes and serum folate measurements. Hematological indexes were analyzed according to supplementation group using multiple regression models that controlled for baseline values (Table 4). At days 14 and 28, the mean PCV was higher in the folic acid supplementation group than in the placebo group; the difference was statistically significant $(P=0.02)$ at day 14 . No other differences in hematological indexes were detected between the two treatment groups.

Serum folate concentrations were measured in a randomly selected sample of 20 children in the placebo group and 49 children in the folic acid group. At baseline, the median serum folate concentrations were similar: $13.8 \mathrm{ng} / \mathrm{mL}$ (range, $7.5-56.1 \mathrm{ng} / \mathrm{mL}$ ) in the folic acid group and $15.0 \mathrm{ng} / \mathrm{mL}$ (range, 4.5-61.0 ng/mL) in the placebo group. At day 14, the median was higher among children who had received folic acid $(17.7 \mathrm{ng} / \mathrm{mL}$; range, $9.1-46.8 \mathrm{ng} / \mathrm{mL})$ than in children who had received placebo (13.4 ng/mL; range, 5.4$18.7 \mathrm{ng} / \mathrm{mL}$ ). No serum folate levels were below the lower bound of normal $(<2.5 \mathrm{ng} / \mathrm{mL})$ in any child at baseline or at day 14 .

Adverse events. Six serious AEs (SAEs), including two deaths, occurred in children who had received folic acid, and 10 SAEs with three deaths in children who had received placebo $(P=0.32)$. In addition to the three early deaths considered in the section on treatment failures, there was one late death from marasmus and pneumonia in a child who had received folic acid and one from the complications of measles in a child who had received placebo. SAEs were mainly hospital admissions with an acute infection such as measles or pneumonia; none were thought to be drug related. Nonserious AEs and other clinical features assessed at days 7, 14, 
TABLE 2

Proportions of patients with parasitemia by treatment group

\begin{tabular}{|c|c|c|c|c|}
\hline Day of study & & Folate & Placebo & $P$ value* \\
\hline \multirow[t]{4}{*}{0} & $n$ & 91 & 93 & \\
\hline & Median parasite density & 25,100 & 31,200 & \\
\hline & Range & $1,300-366,600$ & $1,300-987,600$ & \\
\hline & GM (95\% CI) & $26,000(19,600-34,700)$ & $23,200(18,500-34,400)$ & \\
\hline \multirow[t]{4}{*}{1} & $n / N(\%)$ & $80 / 89(90 \%)$ & $78 / 92(85 \%)$ & 0.37 \\
\hline & Median parasite density & 9,300 & 8,700 & \\
\hline & Range & $30-285,300$ & $100-1,013,000$ & \\
\hline & $\mathrm{GM}(95 \% \mathrm{CI})$ & $8,900(5,900-13,400)$ & $8,200(5,500-12,300)$ & \\
\hline \multirow[t]{3}{*}{2} & $n / N(\%)$ & $47 / 89(53 \%)$ & $42 / 89(47 \%)$ & 0.55 \\
\hline & Median parasite density & 900 & 600 & \\
\hline & Range & $40-87,200$ & $50-46,800$ & \\
\hline \multirow[t]{3}{*}{3} & $n / N(\%)$ & $15 / 90(17 \%)$ & $4 / 89(4 \%)$ & 0.013 \\
\hline & Median parasite density range & 1,000 & 200 & \\
\hline & & $40-17,400$ & $50-900$ & \\
\hline \multirow[t]{3}{*}{7} & $n / N(\%)$ & $4 / 90(4 \%)$ & $0 / 86(0 \%)$ & 0.12 \\
\hline & Median parasite density Range & 2,000 & - & \\
\hline & & $70-40,900$ & - & \\
\hline \multirow[t]{3}{*}{14} & $n / N(\%) \dagger$ & $5 / 86(6 \%)$ & $2 / 83(2 \%)$ & 0.44 \\
\hline & Median parasite density Range & 600 & 1,000 & \\
\hline & & $300-5,200$ & $400-1,500$ & \\
\hline \multirow[t]{3}{*}{28} & $n / N(\%) \ddagger$ & 9/71 (13\%) & $13 / 74(16 \%)$ & 0.49 \\
\hline & Median parasite density Range & 5,000 & 2,500 & \\
\hline & & $400-34,600$ & $100-6,200$ & \\
\hline
\end{tabular}

Based on Fisher exact test.
Based on msp2 typing all considered to be re-infections.

$\doteqdot$ Based on msp2 typing all considered to be re-infections except for one recrudescence in the folic acid treatment group.

and 28 were comparable in the two study groups (data not shown).

\section{DISCUSSION}

To treat malarial anemia, effective drugs are needed that will rapidly eliminate the malaria parasite, and hematinics such as folate and iron may be needed to boost hematological recovery, thus reducing reliance on blood transfusions. ${ }^{14}$ There is some evidence to support the use of iron supplementation $^{2,15}$ in African children with malaria anemia but little to

TABLE 3

Proportions of children treated with SP with parasitemia and parasite densities by supplementation group at various days of follow-up

\begin{tabular}{|c|c|c|c|c|}
\hline $\begin{array}{l}\text { Day of } \\
\text { study }\end{array}$ & & Folate & Placebo & $\begin{array}{c}P \\
\text { value }\end{array}$ \\
\hline \multirow[t]{3}{*}{0} & $\mathrm{n}$ & 45 & 46 & \\
\hline & $\begin{array}{l}\text { Median parasite } \\
\text { density }\end{array}$ & 33,200 & 31,700 & \\
\hline & Range & $2,800-366,600$ & $1,400-98,800$ & \\
\hline \multirow[t]{3}{*}{3} & $n / N(\%)$ & 11/44 (25\%) & 1/44 (2\%) & 0.003 \\
\hline & $\begin{array}{l}\text { Median parasite } \\
\text { density }\end{array}$ & 1,000 & 90 & \\
\hline & Range & $100-17,400$ & & \\
\hline \multirow[t]{3}{*}{7} & $n / N(\%)$ & $3 / 42(7 \%)$ & $0 / 42$ & 0.12 \\
\hline & $\begin{array}{l}\text { Median parasite } \\
\text { density }\end{array}$ & 3,600 & & \\
\hline & Range & $300-40,900$ & & \\
\hline \multirow[t]{3}{*}{14} & $n / N(\%)$ & $5 / 40(13 \%)$ & $1 / 40(3 \%)$ & 0.44 \\
\hline & $\begin{array}{l}\text { Median parasite } \\
\text { density }\end{array}$ & 600 & 1,500 & \\
\hline & Range & $300-5,200$ & & \\
\hline \multirow[t]{3}{*}{28} & $n / N(\%)$ & $7 / 36(20 \%)$ & $11 / 36(30 \%)$ & 0.49 \\
\hline & $\begin{array}{l}\text { Median parasite } \\
\text { density }\end{array}$ & 3,200 & 4,900 & \\
\hline & Range & $400-3,500$ & $400-62,00$ & \\
\hline
\end{tabular}

support treatment with folic acid, although this is frequently given.

In this study, we found that administration of folic acid in a dose of $1 \mathrm{mg} / \mathrm{d}$ for 14 days to children with malaria anemia resulted in higher mean PCV values 14 and 28 days after treatment than seen in children who had received placebo. Although the differences between groups were only modest (an increase in PCV of $\sim 1 \%$ ), the difference between groups at day 14 was statistically significant. This effect was seen despite the fact that initial serum folate levels were within the normal range in all of the randomly selected sample of children who were tested. In a study in Kenya ${ }^{10}$ in which larger doses of folic acid were used, no increase in the proportion of children with a hemoglobin greater than $8 \mathrm{~g} / \mathrm{dL}$ was seen, but mean hemoglobin values were not given. This difference may be caused in part by the fact that subjects in the Kenyan study were older (average age, 80 months) than those in the current trial and to the fact that they were also given iron supplementation.

TABLE 4

Mean values for haematological indices and adjusted mean differences at follow-up by supplementation and group

\begin{tabular}{|c|c|c|c|c|c|c|}
\hline \multirow[b]{2}{*}{ Measurement } & \multicolumn{2}{|r|}{ Folate } & \multicolumn{2}{|r|}{ Placebo } & \multirow{2}{*}{$\begin{array}{c}\text { Adjusted mean } \\
\text { difference }(95 \% \mathrm{CI}) *\end{array}$} & \multirow{2}{*}{$\begin{array}{c}P \\
\text { value }\end{array}$} \\
\hline & $n$ & Mean (SD) & $n$ & Mean (SD) & & \\
\hline \multicolumn{7}{|c|}{ Packed cell volume (\%) } \\
\hline Day 7 & 88 & $21.9(3.1)$ & 85 & $21.4(3.7)$ & $0.2(-0.8,1.2)$ & 0.69 \\
\hline Day 14 & 86 & $27.8(3.4)$ & 80 & $26.4(3.7)$ & $1.2(0.2,2.2)$ & 0.02 \\
\hline Day 28 & 71 & $31.5(3.2)$ & 78 & $30.6(3.8)$ & $0.8(-0.3,1.9)$ & 0.16 \\
\hline \multicolumn{7}{|c|}{ Mean corpuscular volume (fl) } \\
\hline Day 14 & 72 & $83.3(8.4)$ & 73 & $85.4(9.5)$ & $-2.2(-5.1,0.6)$ & 0.12 \\
\hline Day 28 & 54 & $83.5(6.7)$ & 58 & $81.2(8.5)$ & $2.6(0.32,5.2)$ & 0.08 \\
\hline \multicolumn{7}{|c|}{ Reticulocyte index } \\
\hline Day 14 & 80 & 3.6 & 78 & 3.3 & $0.1(-1.6,1.2)$ & 0.21 \\
\hline
\end{tabular}


The results of our trial confirm those of previous studies that have shown that co-administration of folic acid can compromise the efficacy of SP. Differences in the prevalence of parasitemia between children who had received folic acid and those who had received placebo were seen on days 3, 7, and 14 after the start of treatment, but only the difference seen on day 3 was statistically significant. In the Kenyan study, the inhibitory effect of folic acid was also most marked in the first week after the start of treatment. ${ }^{10}$ At day 28 , prevalences of parasitemia had increased in each group and were similar. Molecular typing suggested that, surprisingly, nearly all of these late parasitemias were caused by re-infections; only one unequivocal recrudescence was detected. It is possible that ability of SP to clear parasites may be more susceptible to the inhibitory effect of exogenous folate than its prophylactic effect and, if this is the case, this could explain these findings.

The overall failure rate of SP in the study population was only modest ( $8 \%$ parasitological failure at day 14), and it is possible that a more marked inhibitory effect would have been seen in a population with a higher prevalence of resistant parasites. Folic acid treatment had no impact on parasite clearance in children treated with AP, although this combination contains the anti-folate drug proguanil. This supports the idea that proguanil achieves its synergistic action with atovaquone through an alternative mechanism from inhibition of the folate pathway.

A major limitation of this study was the small sample size. This study was designed to have 305 subjects in each arm, but this figure could not be reached, largely because of a drought in Zambia during the study. The sample size was only one third of that required to detect a $10 \%$ difference in failure rates between the study groups with $95 \%$ confidence. Thus, caution is needed in interpreting our results, but these are consistent with those of other recent studies.

In conclusion, this study has shown that folic acid treatment had a modest effect on hematological recovery in children with malaria anemia and that it had some antagonistic effect on treatment with SP, but not with AP. Neither effect was marked but folic acid is probably best avoided, at least in large doses, when SP is used to treat malaria unless there are specific indications why it should be used.

Received December 14, 2005. Accepted for publication February 13, 2006.

Acknowledgments: We thank the patients and families who participated in this study, the staff and management of Macha Mission Hospital for cooperation, and the local chiefs for support. We are grateful to Dr. Emmanuel Kafwembe, Director of Tropical Diseases Research Centre, Ndola, Zambia, for support. The authors thank the staff of the Dunn Nutrition Unit, Cambridge, for the folate measurements and the members of the Data and Safety Monitoring Board (Prof. Winstanley, Prof. Cousens, and Dr. Mukunyandela) for time and advice. We also thank Dr. Kaur for undertaking the HPLC analyses. Malarone and matching placebo were provided by from
GlaxoSmithKline, and SP, folic acid, and matching placebos by Cosomos Pharmaceuticals. GSK provided financial support for the field work in Zambia. M.M. received a research-training grant from UNDP/World Bank/WHO Special Programme for Research and Training in Tropical Diseases (TDR). The Gates Malaria Partnership supports B.M.G.

Authors' addresses: Modest Mulenga and Phidelis Malunga, Tropical Diseases Research Centre, PO Box 71769, 111 Ndola, Zambia. Steve Bennett (deceased), Caroline Shulman, Katherine Fielding, and Brian Greenwood, London School of Hygiene and Tropical Medicine, Keppel St. London WC1E 7HT, UK. Phillip Thuma, Malaria Institute at Macha (MIAM), PO Box 630166, Choma, Zambia, E-mail: brian.greenwood@lshtm.ac.uk.

\section{REFERENCES}

1. Biemba G, Dolmans D, Thuma PE, Weiss G, Gordeuk VR, 2000. Severe anaemia in Zambian children with Plasmodium falciparum malaria. Trop Med Int Health 5: 9-16.

2. Gera T, Sachdev HP, 2002. Effect of iron supplementation on incidence of infectious illness in children: systematic review. $\mathrm{Br}$ Med J 325: 1142-1144.

3. Menendez C, Fleming AF, Alonso PL, 2000. Malaria-related anaemia. Parasitol Today 16: 469-476.

4. Fleming AF, 1981. Haematological manifestations of malaria and other parasitic diseases. Clin Haematol 10: 983-1011.

5. Abdalla SH, 1990. Iron and folate status in Gambian children with malaria. Ann Trop Paediatr 10: 265-272.

6. Watkins WM, Sixsmith DG, Chulay JD, Spencer HC, 1985. Antagonism of sulfadoxine and pyrimethamine anti-malarial activity in vitro by $\mathrm{p}$ - aminobenzoic acid, p-aminobenzoylglutamic acid and folic acid. Mol Biochem Parasitol 14: 55-61.

7. Oppenheimer SJ, Cashin P, 1986. Serum and red cell folate levels associated with malarial parasitaemia. Trans $R$ Soc Trop Med Hyg 80: 169-177.

8. Das KC, Virdi JS, Herbert V, 1992. Survival of the dietary deprived: folate deficiency protects against malaria in primates. Blood 80: 281a.

9. Boele van-Hensbroek M, Morris-Jones S, Meisner S, Jaffar S, Bayo L, Dackour R, Phillips C, Greenwood BM, 1995. Iron, but not folic acid, combined with effective anti-malarial therapy promotes haematological recovery in African children after acute falciparum malaria. Trans $R$ Soc Trop Med Hyg 89: 672-676.

10. Carter JY, Loolpaptit MP, Lema OE, Tome JL, Nagelkerke NJD, Watkins WM, 2005. Folic acid supplementation reduces the efficacy of antifolate anti-malarial therapy. Am J Trop Med Hyg 73: 166-170.

11. Dzinjalamala FK, Macheso A, Kublin JG, Taylor TE, Barnes KI, Molyneux ME, Plowe CV, Smith PJ, 2005. Blood folate concentrations and in vivo sulfadoxine-pyrimethamine failure in Malawian children with uncomplicated Plasmodium falciparum malaria. Am J Trop Med Hyg 72: 267-272.

12. World Health Organization, 2000. Severe falciparum malaria. Trans R Soc Trop Med Hyg 94 (Suppl 1): S1-S90.

13. Snounou G, 2002. Genotyping of Plasmodium spp. nested PCR. Methods Mol Med 72: 103-116.

14. Ekval H, 2003. Malaria and anemia. Curr Opin Hematol 10: 108114.

15. Verhoeff FH, 2001. Iron Deficiency and Malaria as Determinants of Anaemia in African Children. Universal Press: Veenendaal, The Netherlands. 